\title{
Brain Impairment
}

http://journals.cambridge.org/BIM

Additional services for Brain Impairment:

Email alerts: $\underline{\text { Click here }}$

Subscriptions: $\underline{\text { Click here }}$

Commercial reprints: $\underline{\text { Click here }}$

Terms of use : $\underline{\text { Click here }}$

\section{Planning Ability Following Moderate to Severe Traumatic Brain Injury: Performance on a 4-Disk Version of the Tower of London}

David Shum, Hannah Gill, Miranda Banks, Annick Maujean, Janelle Griffin and Heather Ward

Brain Impairment / Volume 10 / Issue 03 / December 2009, pp 320 - 324

DOI: 10.1375/brim.10.3.320, Published online: 21 February 2012

Link to this article: http://journals.cambridge.org/abstract S1443964600001935

\section{How to cite this article:}

David Shum, Hannah Gill, Miranda Banks, Annick Maujean, Janelle Griffin and Heather Ward (2009). Planning Ability Following Moderate to Severe Traumatic Brain Injury:

Performance on a 4-Disk Version of the Tower of London. Brain Impairment, 10, pp 320-324 doi:10.1375/brim.10.3.320

Request Permissions : $\underline{\text { Click here }}$ 


\title{
Planning Ability Following Moderate to Severe Traumatic Brain Injury: Performance on a 4-Disk Version of the Tower of London
}

\author{
David Shum, ${ }^{1,2}$ Hannah Gill, ${ }^{3}$ Miranda Banks, ${ }^{2}$ Annick Maujean, ${ }^{2}$ Janelle Griffin, ${ }^{4}$ \\ and Heather Ward ${ }^{5}$ \\ ${ }^{1}$ Griffith Institute for Health and Medical Research, Griffith University, Brisbane, Australia \\ 2 School of Psychology, Griffith University, Brisbane, Australia \\ ${ }^{3}$ School of Health and Rehabilitation Sciences, University of Queensland, Australia \\ ${ }^{4}$ Brain Injury Rehabilitation Unit, Occupational Therapy Department, Princess Alexandra Hospital, \\ Brisbane, Australia. \\ ${ }^{5}$ Albany Creek Specialists Centre, Albany Creek, Brisbane, Australia
}

\begin{abstract}
This study aimed to assess planning ability in adults with traumatic brain injury (TBI) using a 4-disk version of the Tower of London (TOL). Thirty three individuals with TBI were compared with equivalent numbers of matched controls. Overall, the TOL 4 was shown to be sensitive to the effects of brain injury, with the TBI group performing significantly more poorly on this version of the planning test than the matched controls. More specifically, group differences were found to be related to the complexity of the planning problems, particularly among a TBI subgroup with localised prefrontal damage. Results of the study provide support for the adverse effects of TBI on planning ability, and the important role of the prefrontal cortex in planning.
\end{abstract}

Keywords: planning, Tower of London, adults, TBI

Impairment in planning ability, a component of executive functioning, is commonly reported in individuals following traumatic brain injury (TBI). Planning is a complex process that involves goal formation, development of a sequence of strategies to fulfil that goal, implementation of the devised strategies, and the ability to utilise feedback to alter unsuccessful strategies (Kaller, Unterraniner, Rahm, \& Halsband, 2004). It is generally understood that impaired planning might reflect damage within the prefrontal cortex, which is particularly susceptible to trauma. Neuroimaging studies of individuals with TBI (e.g., Levin, et al., 1997a) identified a rostrocaudal gradient of lesion prevalence, with the frontal region being the most frequent lesion site. Unfortunately, impairment in planning ability has significant functional consequences for individuals with TBI because this ability is essential for independent living (Jefferson, Paul, Ozonoff, \& Cohen, 2006).

The original 3-disk Tower of London (TOL3), developed by Shallice (1982), is one of the most widely used tests of planning ability (Anderson, Anderson, \& Lajoie, 1996; Baker, Segalowitz, \& Ferlisi, 2001; Cockburn, 1995; Strauss, Sherman, \& Spreen, 2006). Three coloured disks are presented on three rods of different heights. The test taker is required to rearrange them within $60 \mathrm{~s}$ and in as few moves as possible to match a target configuration of the disks. The minimum number of moves increases from 2 to 5 across the 12 items of the task. According to Shallice, successful performance on the TOL3 requires the ability to: (a) formulate an overall schema, (b) identify the subgoals required for the schema and arranging them into a sequence 
of moves, and (c) hold the subgoals and overall schema in spatial working memory.

Despite its popularity, research employing the TOL3 to assess planning ability in individuals with TBI has not found this test to be sensitive. For example, Cockburn (1995) compared 20 adults with severe TBI (aged 18 to 62 years) and 20 controls on the TOL3 and two other tests of executive function (viz., a modified version of the Wisconsin Card Sorting Test and the Controlled Oral Word Association Test). While significant group differences were found for the other two tests, such a difference was absent for the TOL3. Similarly, Ponsford and Kinsella (1992) were unable to distinguish between 22 adults with severe TBI (aged 16 to 43 years) and 19 controls (matched on age and years of education) on the number of TOL3 problems solved on the first attempt. In a more recent fMRI study, Rasmussen et al. (2006) showed that although different brain activation patterns were found for a group of adults with severe TBI and a group of matched controls while they were undertaking the TOL3, no differences were found between the two groups on their TOL3 scores. One possible reason for these findings is the heterogeneous nature (e.g., in terms of severity, location of localised lesions) of the TBI samples in these studies.

In studies that used the TOL3 to assess planning in children with TBI, Levin et al. (1994) found that young children (6 to 10 years) with TBI performed significantly more poorly than controls. However, this impairment was not found for older children (11 to 16 years) with TBI. Similarly, in a later study by Levin et al. (1997b) that analysed cross-sectional and longitudinal data from children with TBI (aged 5 to 18 years), the TOL3 was found to be sensitive to TBI in younger but not older children. These authors argued that the differential effect of TBI on planning in children is likely to be due to a ceiling effect of the TOL3 for the older children. To clarify the effects of TBI on older children and adults, they suggested that the range of difficulty of the TOL be increased.

A number of researchers (e.g., Anderson et al., 1996; Culbertson \& Zillmer, 1998; Krikorian, Bartok, \& Gay, 1994) have attempted to increase the difficulty and sensitivity of the TOL3 by modifying its materials and/or administration rules and scoring method. Tunstall (1999) has tried to do the same by developing a 4-disk version of the test (viz., TOL4). However, unlike the others, she applied rigorous psychometric criteria for all stages of test development (e.g., problem design, item selection, item testing, evaluation of reliability and validity). She initially created a large pool of possible items that varied in the number of moves required to solve, with the fewest possible alternative solution paths. After testing the items on a sample of children and adults, the most sensitive items were selected from the pool using item discrimination and difficulty. Scoring on the TOL4 was adapted from Krikorian et al.'s method which involves removing possible speed and accuracy confounds, and giving maximum potential for discrimination by including 3 trials per item. There were 10 items altogether for TOL4 5 'Simple' (2 to 5 moves) and 5'Complex' (6 to 9 moves) problems. Tunstall also changed the disk colours from those used in the TOL3 to avoid the necessity for colour-vision screening. Moreover, she gathered normative data from 334 children aged 5 to 15 years and 113 adults aged 17 to 53 years to allow the test to be used quantitatively in clinical assessments. Tunstall's TOL4 has been found to have adequate test-retest reliability (.71), to load on the same factor as the Porteus Mazes, another test of planning ability, and to be sensitive in detecting planning impairments in both children (6-10 years) and adolescents (11-16 years) with TBI (Shum, et al., 2000). To date, however, the TOL4 has not been used in adults with TBI.

The present study aimed to employ a more sensitive version of the TOL to assess planning ability in adults with TBI. Tunstall's (1999) TOL4 was chosen for this purpose because of its increased level of test difficulty, its improved scoring method, and its promising psychometric properties. Given that impairments in planning ability are linked to damage to the prefrontal cortex, the present study also aimed to explore the 'prefrontal' model of planning by comparing the TOL 4 performance of TBI individuals with focal prefrontal damage with those with non prefrontal damage and with controls. Indeed some of the results reported in the literature could be confounded by the heterogeneous nature of the TBI group. In the present study, one source of heterogeneity of the TBI group was controlled by grouping them according into 'prefrontal' and 'non-prefrontal' damage.

Generally, it was hypothesised that the TOL4, particularly the set of five Complex items (i.e., those with more than 5 moves), would be sensitive to the effect of TBI in adults. More specifically, it was hypothesised that:

1. TBI patients with prefrontal damage would perform more poorly than matched controls on the Complex but not the Simple items of the TOL4.

2. TBI patients without prefrontal damage would not perform significantly different than matched controls on either the Complex or Simple Items of the TOL4 


\section{Method}

\section{Participants}

The TBI group comprised 33 adults ( 25 males) who were no longer in post traumatic amnesia (PTA). They were recruited from the Commonwealth Rehabilitation Service and the Brain Injury Rehabilitation Unit at the Princess Alexandra Hospital, Brisbane. The average age of this group was 32.52 years $(S D=9.98$ years, range $=19-53$ years) and the average number of years of education was 12.42 years $(S D=2.60$ years, range $=8$ 21 years). The Glasgow Coma Scale scores (GCS: Teasdale \& Jennett, 1974) of the participants with TBI ranged from 3 to 15 at the scene of injury, with mean GCS of $7.68(S D=3.68)$. The average length of PTA was 57.60 days ( $S D=55.96$ days, range $3-183$ days). The average time since injury was 288.76 days $(\mathrm{SD}=214.58$ days, range $=30$ 788 days). Using length of PTA as a measure of severity, all these participants had moderate to severe TBI.

Based on results of CT scan, the TBI group was divided into two subgroups: a subgroup that comprised 15 individuals with localised prefrontal damage (viz., prefrontal) and a subgroup that comprised 16 individuals without localised prefrontal damage (viz., non prefrontal). Two participants were not classified into either subgroup because of missing data. The two subgroups were not found to be significantly different in injury severity as measured by length of PTA $(p>.05)$.

The control group was the same size with equivalent gender ratio as the TBI group. To provide match comparison (in terms of age, gender and years of education) for the TBI prefrontal and non-prefrontal subgroups, two subgroups of controls were also formed. Participants in this group were recruited from the general community in Brisbane. None had a history of TBI or other neurological disorders. Their average age was 31.76 years $(S D=9.70$ years, range $=18-49$ years $)$, and the average number of years of education was 12.64 years $(S D=1.90$ years, range $=9-16$ years $)$. There were no significant differences between the TBI and control groups on age, $t(64)=-.31, p=$ 0.75 , or years of education, $t(64)=.38, p=0.71$. All participants were from English-speaking backgrounds. Both the TBI and control groups were part of a larger study that included assessment of prospective memory and executive functions.

\section{Apparatus}

Tunstall's (1999) TOL4 comprises four wooden coloured disks (black, blue, white, \& yellow) and three wooden rods mounted on a rectangular wooden block. The three rods could hold a maximum of two, three and four disks respectively. As with the TOL3, the objective of the TOL4 is to rearrange the coloured disks within a specified number of minimum moves from a starting configuration to match a target configuration. The TOL4 has 10 items, with 2, 3, 5, 5, 6, 6, 6, 7, 9, moves respectively. All items begin with the same starting configuration. The five items requiring 2 to 5 moves are considered 'Simple' and the five items requiring 6 to 9 moves are considered 'Complex'. The scoring of the TOL4 is based on the method developed by Krikorian et al. (1994) for the TOL3, whereby instead of using the number of correct problems solved within a time limit, the time limit is removed and participants are given three attempts for each item. Three points are awarded for solving an item on the first trial, two points on the second trial, one point on the third trial, and zero if the problem is not solved within three trials. Removing the time limit eliminates a possible psychomotor-speed confound. Planning time (see below) was nonetheless measured in this study to allow for an additional measure.

Measures obtained for the TOL4 were the total scores for all the items and total scores for the Simple and Complex items. Other measures included the average initial planning time (i.e., the time between the target configuration being shown and the first disk being picked up and released in the first trial) for all the items as well as the average initial planning time for the Simple and Complex items.

\section{Procedure}

After informed consent was obtained, all participants were administered the TOL4 individually using standardised materials and instructions. On average, administration of the TOL4 took approximately 15 min and it was included as part of a larger study. Ethical approvals were obtained from the two organisations where the participants were recruited and from Griffith University.

\section{Results}

Table 1 summarises the performance of the TBI and control groups on the total, Simple total and Complex total scores on the TOL4. Results of an independent samples $t$ test indicate that participants with TBI obtained significantly lower total scores than the controls $(t[64]=2.52, p=.01$, $d=0.62)$. There was no significant group difference on the total Simple score $(t[64]=1.22$, $p=.23, d=0.31$ ) but participants in the TBI group obtained significantly lower scores on the 


\section{TABLE 1}

Means and Standard Deviations of TOL 4 Scores for the TBI and Control Groups

\begin{tabular}{lrrrr} 
Scores & \multicolumn{2}{c}{ TBI } & \multicolumn{2}{c}{ Controls } \\
& \multicolumn{2}{c}{$(n=33)$} & \multicolumn{2}{c}{$(n=33)$} \\
& $M$ & $S D$ & \multicolumn{1}{c}{$M$} & \multicolumn{1}{c}{$S D$} \\
\hline Total $(\max =30)$ & 21.42 & 3.00 & 23.06 & 2.22 \\
Simple $(\max =15)$ & 14.42 & 0.97 & 14.67 & 0.60 \\
Complex $(\max =15)$ & 6.97 & 2.89 & 8.39 & 2.11 \\
Total Time & 11.26 & 5.16 & 11.13 & 6.19 \\
Simple Time & 5.95 & 3.45 & 5.10 & 1.61 \\
Complex Time & 16.57 & 8.16 & 17.17 & 11.65 \\
\hline
\end{tabular}

Complex total score than the controls $(t[64]=$ $2.29, p=.03, d=0.56$ ). No significant group differences were found for the average planning time for the Simple, Complex, and full item sets.

The performance of the two TBI subgroups (viz., prefrontal and non-prefrontal) and their respective matched controls on the TOL4 are summarised in Table 2. Participants in the prefrontal subgroup were found to have significantly lower total and Complex total scores than the controls $(t[28]=2.26, p=.03, d=0.82 ; t[28]=2.18$, $p=.04, d=0.80$ ), but the two groups were not found to be different on the Simple total scores $(t[28]=1.08 ., p=.29, d=0.39)$. Participants in the non prefrontal group were not found to be different from the controls on either total $(t[30]=0.36$, $p=.85, d=0.13)$, Simple total $(t[30]=0.66$, $p=.52, d=0.24)$, or Complex total scores $(t[30]=0.22, p=.83, d=0.08)$. No significant group differences were found between the two TBI subgroups and their respective controls on the average planning time either for the Simple, Complex, and full item sets.

\section{Discussion}

Results of this study indicate that as a measure of planning ability, the Complex total score of the TOL4 is sensitive to the effects of moderate to severe TBI in adults. This finding is in contrast to those reported by Cockburn (1995), Ponsford and Kinsella (1992), and Rasmussen et al. (2006) who used the TOL3 to assess planning in individuals with TBI. As mentioned in the introduction, the absence of significant group differences in the previous studies is likely to be due to the ceiling effects of the TOL 3 for older children and adults (Levin et al., 1994; Levin et al., 1997b). By including items that involve more than 5 moves (viz., 6, 7 and 9), the TOL4 used in this study has extended the difficulty of the TOL. This, in turn, has improved the sensitivity of the TOL as a measure of planning ability and showed that individuals with moderate to severe TBI are impaired on planning ability. This finding corroborates the results reported by Shum et al. (2000) who used the same TOL4 and showed that planning is impaired in older children with TBI. In addition, this finding suggests that clinicians who work with individuals with TBI should consider using this more sensitive version of TOL to assess planning ability. Most importantly, the finding of this study clarifies the nature of planning impairment in individuals with TBI. While these individuals do not seem to have a problem in carrying out comparatively simple plans, they show difficulty in forming and executing complex plans that require considerable cognitive resources.

Interesting results were also obtained when the TBI group was subdivided into the prefrontal and non-prefrontal subgroups. While the former was found to be impaired on the total and Complex total scores of the TOL4 ), the latter's performance on this test was found to be compa-

TABLE 2

Means and Standard Deviations of TOL 4 Scores for Four Groups of Participants

\begin{tabular}{|c|c|c|c|c|c|c|c|c|}
\hline \multirow[t]{4}{*}{ Scores } & \multicolumn{8}{|c|}{ Localised Prefrontal Damage } \\
\hline & \multicolumn{4}{|c|}{ Yes } & \multicolumn{4}{|c|}{ No } \\
\hline & \multicolumn{2}{|c|}{$\mathrm{TBI}(n=15)$} & \multicolumn{2}{|c|}{ Controls $(n=15)$} & \multicolumn{2}{|c|}{$\mathrm{TBI}(n=16)$} & \multicolumn{2}{|c|}{ Controls $(n=16)$} \\
\hline & M & $S D$ & $M$ & $S D$ & M & $S D$ & M & $S D$ \\
\hline Total $(\max =30)$ & 21.07 & 2.87 & 23.20 & 2.27 & 22.31 & 2.73 & 22.63 & 2.09 \\
\hline Simple $(\max =15)$ & 14.47 & 1.13 & 14.80 & 0.41 & 14.31 & 0.87 & 14.50 & 0.73 \\
\hline Complex $(\max =15)$ & 6.60 & 2.38 & 8.40 & 2.13 & 7.94 & 2.74 & 8.13 & 2.06 \\
\hline Total Time & 10.91 & 4.64 & 10.65 & 7.29 & 11.14 & 5.89 & 11.04 & 5.15 \\
\hline Simple Time & 6.38 & 2.84 & 5.27 & 2.05 & 5.66 & 4.14 & 5.13 & 1.12 \\
\hline Complex Time & 15.44 & 8.06 & 16.03 & 13.21 & 11.41 & 5.89 & 11.03 & 5.15 \\
\hline
\end{tabular}


rable to the matched controls. These results support the important role of the prefrontal cortex in planning and highlight the effects of localised prefrontal damage on planning ability following TBI.

There was no injury or site of injury effects on the average planning time of the TOL4. This suggests that compared to the total scores which measure accuracy, average planning time is not a sensitive measure of planning ability, possibly because the time taken between being shown the target configuration and making the first move may not be a 'true' measure of planning ability. For instance, taking a longer time to make the first move, especially in the case of those with TBI, could be due to a generalised or specific psychomotor processing speed deficit or a lack of confidence. Alternatively, taking a shorter time to make the first move could have resulted from impulsivity. Therefore, average TOL4 planning time, unless it can be measured more precisely or broken down appropriately, appears not to be very informative for a person's planning ability.

There are a number of limitations associated with this study. First, the TBI sample is a convenient one and relatively small and heterogeneous (e.g., in terms of severity of injury, time since injury). Therefore, the results obtained should be replicated before they can be generalised. Second, allocation of the TBI participants into the prefrontal and non-prefrontal subgroups was based on general CT findings rather than detailed volumetric analysis of CT or MRI images. Thus, the allocation may not be precise and be subjected to error. Third, no measures or external ratings of planning were included in the study to confirm that the TOL4 is related to these measures or ratings.

To further explore whether abilities measured by the TOL 3 and TOL4 are quantitatively or qualitatively different, fMRI can be used to identify the cortical area(s) involved while undertaking these tests. Future studies can also extend the utility of the TOL4 in assessing planning ability in other clinical populations by comparing these samples with matched controls.

\section{References}

Anderson, P., Anderson, V., \& Lajoie, G. (1996). The Tower of London test: Validation and standardization for pediatric populations. The Clinical Neuropsychologist, 10, 54-65.

Baker, K., Segalowitz, S. J., \& Ferlisi, M. (2001). The effect of differing scoring methods for the Tower of London task on developmental patterns of performance. The Clinical Neuropsychologist, 15, 309313.
Cockburn, J. (1995). Performance on the Tower of London test after severe head injury. Journal of International Neuropsychological Society, 1, 537-544.

Culbertson, W. C., \& Zillmer, E. A. (1998). The construct validity of the Tower of London DX as a measure of the executive functioning of ADHD. Assessment, 5, 215-226.

Jefferson, A. L., Paul, R. H., Ozonoff, A., \& Cohen, R. A. (2006). Evaluating elements of executive functioning as predictors of instrumental activities of daily living (AIDLS). Archives of Clinical Neuropsychology, 21, 311-320.

Kaller, C. P., Unterrainer, J. M., Rahm, B., \& Halsband, U. (2004). The impact of problem structure on planning: Insights from the Tower of London task. Cognitive Brain Research, 20, 462-472.

Krikorian, R. Bartok, J., \& Gay, N. (1994). Tower of London procedure: A standard method and developmental data. Journal of Clinical and Experimental Neuropsychology, 16, 840-850.

Levin, H. S., Mendelsoh, D., Lily, M. A., Fletcher, J. M., Culhane, K. A., Chapman, S. B., et al. (1994). Tower of London performance in relation to magnetic resonance imaging following closed head injury in children. Neuropsychology, 8, 171-179.

Levin, H. S., Mendelsohn, D., Lily, M. A., Yeakley, J., Song, J., Scheibell, R. S., et al. (1997a). Magnetic resonance imaging in relation to functional outcome of pediatric closed head injury: A test of the OmmayaGennarelli Model. Neurosurgery, 40, 432-441.

Levin, H. S., Song, J., Scheibel, R. S., Fletcher, J. M., Harward, H., Lilly, M., et al. (1997b). Concept formation and problem-solving following closed head injury in children. Journal of International Neuropsychological Society, 3, 598-607.

Ponsford, J., \& Kinsella, G. (1992). Attention deficits following closed-head injury. Journal of Clinical and Experimental Neuropsychology, 14, 822-838.

Rasmussen, I. A., Antonsen, I. K., Berntsen, E. M., Xu, J., Lagopoulous, J., \& Haberg, A. K. (2006). Brain activation measured using functional magnetic resonance imaging during the Tower of London task. Acta Neuropsychiatrica, 18, 216-225.

Shallice, T. (1982). Specific impairments of planning. Philosophical Transactions of Royal Society of London, Series B, Biological Sciences, 298, 199-209.

Shum, D., Short, L., Tunstall, J., O'Gorman, J. G., Wallace, G., Shephard, K., et al. (2000). Performance of children with traumatic brain injury on a 4-disk version of the Tower of London and the Porteus Maze. Brain \& Cognition, 44, 59-62.

Strauss, E., Sherman, E. M. S., \& Spreen, O. (2006). A compendium of neuropsychological tests (3rd ed.). New York: Oxford University Press.

Teasdale, G., \& Jennett, B. (1974). The Glasgow Coma Scale. Lancet, 2, 81 .

Tunstall, J. (1999). Improving the utility of the Tower of London: A neuropsychological test of planning. Unpublished M Phil: Griffith University, Brisbane, Australia. 